\title{
BETA-DELAYED PROTON DECAY IN THE LANTHANIDE REGION*
}

\author{
J.M. Nitschke, P.A. Wilmarth, J. Gilat, ${ }^{* *}$ and P. Möller*** \\ Lawrence Berkeley Laboratory, University of California, Berkeley, CA 94720 \\ K.S. Toth \\ Oak Ridge National Laboratory, Oaik Ridge, TN 37831
}

\begin{abstract}
A total of 24 new $\beta$-delayed proton precursors and several new decay branches in the region of $56<\mathrm{Z}<72$ and $\mathrm{N}<82$ have been identified with the OASIS on-line mass separator facility at the SuperHILAC in Berkeley. Proton spectra and halflives were measured for all precursorg. Additional properties determined for many of the isotopes include spins and parities of the precursors, final state feedings, proton branching ratios, $\left(Q_{E C}-S_{p}\right)$ energy differences, and new levels in the proton decay daughters. Mixed $\beta$-delayed proton decay from precursor isomers and ground states has been observed. The proton decay has been compared to statistical model calculations using Gamow - Teller (GT) $\beta$-strength functions obtained from RPA calculations with Nilsson model wave functions. For the $\mathrm{N}=81$ precursors ${ }^{151} \mathrm{Yb},{ }^{149} \mathrm{Er}$, and ${ }^{147} \mathrm{Dy}$, pronounced structure in the proton spectra has been observed. An interpretation of this structure in the framework of "doorway" states is proposed.
\end{abstract}

\section{INTRODUCTION}

With the recent discovery of $\beta$-delayed proton emission in europium this decay mode has now been observed in an unbroken sequence of elements from $Z=52$ (Te) to $\mathrm{Z}=71(\mathrm{Lu})$, which shows, as expected, that $\beta$-delayed proton decay becomes a prominent decay mode for nuclei near the proton drip line.

Isotopes with various degrees of deformation from quasi spherical near the closed shells $\mathrm{Z}=\mathbf{5 0}$ and $\mathrm{N}=\mathbf{8 2}$ to highly deformed near $\mathrm{Z}=60$ have been studied. Nuclei in the deformed region show smooth proton spectra that are well described by a statistical model due to the close level spacings at excitation energies that are sufficiently high for proton decay. For the interpretation of our experimental results we have used a statistical model formulated originally by Hornshøj, et al. ${ }^{2}$ with the following modifications first: suggested by Hardy ${ }^{3}$ : the original Gilbert and Cameron level density formula was replaced by a backshifted Fermi gas prescription, and the $\gamma$ width calculation was modified to include the effects of the giant dipole resonance and normalized to experimental photosbsorption cross sections. We also incorporated an additional set of optical model parameters, more appropriate for the calculation of transmission coefficients below the Coulomb barrier ${ }^{4}$. There are several critical parameters that enter into statistical model calculations: the total decay energy $Q_{E C}$, the proton separation energy $S_{p}$, the level density $\rho$, the $\gamma$ decay width $\mathrm{l}_{\gamma}$, the optical model parameters, the spin and parity of the precursor $\mathrm{J}^{\pi}$, and the $\beta$-strength function $\mathrm{S}_{\beta}$. Calculated quantities that can be compared to experiment are: tbe proton energy spectrum, the proton branching ratios to states in the final nucleus, and the total proton $\beta$-decay branching ratio $b_{p}$. There are large variations in the strength with which the various

* This work was supported by the Director, Ofice of Energy Research, Division of Nuclear Physics of the Office of High Energy and Nuclear Physics of the U.S. Department of Energy under Contract DE- AC03-76SF00098 and DE-AC05-84OR21400.

** On leave from Soreq Nuclear Research Center, Yavne 70600, Israel.

*** On leave from Lund University, Sweden. 
input parameters affect the calculated quantities. We have, for example, used the strong coupling between $\mathrm{J}^{*}$ and the final state proton branching ratios to determine the spin and parity of several new precursors listed in Table I. In cases where proton emission occurs both from the ground and isomeric states of the precursor their intensity ratio can also be calculated from 'he final state proton branching ratios. The most profound influence on the results of tatistical model calculations is exercised by $S_{\beta}$. (The only parameters $S_{\beta}$ does not affect strongly are the final state proton branching ratios.) Without detailed knowledge of $S_{\beta}$ either from experiments such as total absorption $\gamma$-ray spectroscopy or from theory it is rarely possible to reproduce the correct centroid and shape of the proton spectrum - simultaneously with $b_{p}$, the half-life, and to some extent the final state proton branching ratios. Since no $S_{\beta}$ measurements in our region of interest are available (except for ${ }^{147 m} \mathrm{Dy}^{6}$ ) we have performed calculations of GT $\beta$-strength functions using deformed Nilsson model wave functions in a modified oscillator potential ${ }^{\top}$. Pairing is treated in BCS approximation. A simple residual interaction with a coupling constant of $\chi_{G T}=23 / \mathrm{A} \mathrm{MeV}$ is added and is treated in the RPA approximation. The strength of this interaction has been adjusted to obtain agreement between the calculated and experimental energies of the giant GT resonance for ${ }^{208} \mathrm{~Pb}$ and ${ }^{144} \mathrm{Sm}$. The Niisson model $\kappa$ - and $\mu$-parameters for the proton and neutron single particle potentials are: $\kappa_{p}=0.0637, \mu_{p}=0.6000, \kappa_{n}=$ 0.0637 , and $\mu_{n}=0.4200$. These parameters have been determined from adjustment of calculated single particle levels to experimental data in the rare earth region ${ }^{8}$. Deformation parameters were taken from Ref. 9 . We have thus far not extended these calculations to isomers.

After a brief discussion of experimental details in section 2, a comparison between experimental and calculated proton spectra is made in section 3 . For precursors near the $N=82$ closed shell the statistical model description breaks down, and $S_{\beta}$ calculations have to be modified. This is discussed in section 4 for a set of three $\mathrm{N}=$ 81 even-odd jsotones.

\section{EXPERIMENTAL}

The isotopes presented here were produced in compound nucleus reactions of neutron-deficient projectiles ( ${ }^{56} \mathrm{Ar},{ }^{40} \mathrm{Ca},{ }^{46} \mathrm{Ti},{ }^{50,52} \mathrm{Cr},{ }^{54,56} \mathrm{Fe},{ }^{38} \mathrm{Ni}$, and ${ }^{64} \mathrm{Zn}$ ) and neutron-deficient targets $\left({ }^{58} \mathrm{Ni},{ }^{90} \mathrm{Zr},{ }^{92,94} \mathrm{Mo}\right.$, and $\left.{ }^{96} \mathrm{Ru}\right)$ at the LBL SuperHILAC. The evaporation residue recoils were stopped in a tantalum catcher operated close to its melting point ( $3000 \mathrm{C}$ ) located inside a surface ionization source. After diffusing out of the catcher and being ionized, the product ions were accelerated to $50 \mathrm{keV}$ and mass separated with the on-line isotope separator OASIS ${ }^{10}$. The separator was calibrated with stable rare earth isotopes introduced into the ion source as minute quantities of the oxides. A single isobaric chain was selected at the focal plane of the separator and transported ionoptically to a shielded, low background spectroscopy laboratory. Here the 50-keV ions were implanted in a 50- $\mu \mathrm{m}$ Mylar tape and transported within $64 \mathrm{~ms}$ to an array of detectors consisting of a Si $\Delta \mathrm{E}-\mathrm{E}$ particle telescope, a HyperPure Ge (HPGe) detector ( $0.7 \mathrm{keV}$ FWHM resolution at $122 \mathrm{keV}$ ), two n-type Ge detectors with relative efficiencies of $24 \%$ (1.9 keV F WHM resolution at $1332 \mathrm{keV}$ ) and $52 \%(2.4$ keV F WHM resolution at $1332 \mathrm{keV}$ ), and a thin plastic $\Delta \mathrm{E}_{\beta}$ detector. A scale drawing of the detector arrangernent is shown in Fig. 1. Coincidences between protons, $x$ rays, $\gamma$ rays, and positrons were recorded event-by-event on magnetic tape for subsequent off-line analysis. After the arrival of a new sample at the detectors, a quariz controlled clock was started to tag all events with a relative time signal for half-life determination. Appropriate counting time intervals were chosen, taking into account the known or expected half-lives. A new batch of collected isotopes was supplied by the computer controlled tape system at the conclusion of each counting interval. Concurrent with 
the evest-by-event data acquisition, time resolved spectroscopy singles data were taken with the 52\% $\gamma$-ray detector and the HPGe detector. Counting intervals for the singles measurements were typically divided into eight equal time bins. Singles data from the $24 \%$ detector were also recorded to correct for summing effects in the $52 \%$ detector. Energy and efficiency calibrations of the Ge detectors were carried out with standard sources; $x$-ray and $\gamma$-ray efficiencies were linked to the particle telescope efficiency via $\alpha$ particles and $\gamma$ rays from ${ }^{241} \mathrm{Am}$ decay.

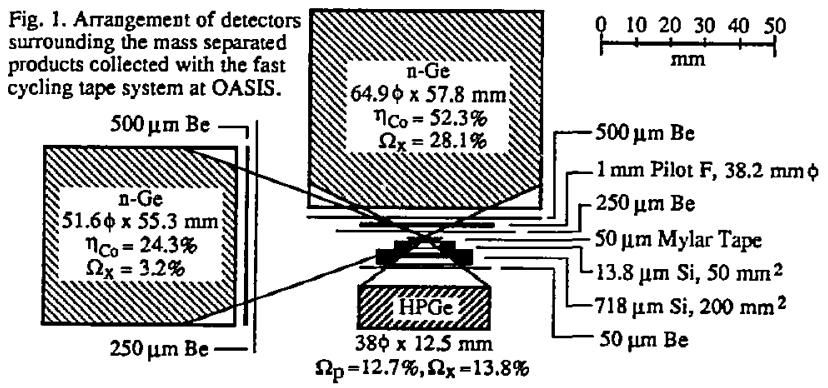

\section{RESULTS: DEFORMED REGION}

Beta-delayed proton decay becomes possible when the total decay energy of a nucleus $Q_{E C}$ exceeds the proton separation energy $S_{p}$ in the $\beta$-decay daughter. The Coulomb barrier prevents proton decay from competing with $\gamma$-decay until a threshold energy $\Theta_{p}$ is exceeded, i.e., $\left(Q_{E C}-S_{p}-\Theta_{p}\right)>0$. In the lanthanide region $\Theta_{p}$ is about 2-3 MeV. Calculations show that the observed protion emission thresholds in this region correspond to a proton decay width $\Gamma_{\mathrm{p}}$ of $10^{-5} \mathrm{eV}$ or a proton-to $\gamma$-width ratio $\Gamma_{p} / \Gamma_{\gamma}$ of $\sim 10^{-45}$, which yields $\Gamma_{\gamma}=\Gamma_{p} \times 10^{4}=0.1 \mathrm{eV}$, equivalent to a $\gamma$ lifetime of $7 \times 10^{-15}$ s. Lifetimes of this order are associated with E1 single-particle transitions of about $0.4 \mathrm{MeV}$ or $\mathrm{Ml}$ transitions of about $1.7 \mathrm{MeV}$, which are typical energies encountered in $\gamma$-ray cascades.

Table 1 gives a summary of all new isotopes and new decay branches observed with OASIS to date. With the exception of ${ }^{142} \mathrm{Dy},{ }^{144} \mathrm{Dy}$, and ${ }^{148} \mathrm{Er}$ all $\beta$-delayed proton precursors, including those known previously, are odd- $N$ nuclei. The question arises why several even- $N$ nuclei that have a lower isospin than known $\beta$-delayed proton precursors in the same isobaric chain have no appreciable proton branches ( for example ${ }_{60}^{128} \mathrm{Nd},{ }_{61}^{131} \mathrm{Pm}$, and ${ }_{65}^{141} \mathrm{~Tb}$ ) even though $\beta$-delayed proton decay is energetically possible. An experimental reason is that in some cases such a decay is difficult to observe because of other strong proton precursors in the same chain. Another reason supported by $S_{\beta}$ calculations, is that most of the available $\beta$ strength may go to levels at excitation energies below $\left(S_{p}+\theta_{p}\right)$.

Rather than superficially discussing several new isotopes listed in Table 1, the state of the present experimental and interpretational capabilities will be illustrated by presenting results for one typical well deformed $\beta$-delayed proton precursor $-{ }_{58}^{123} \mathrm{Ce}$ - in more detail. Fig. 2 shows the measured proton spectrum (histogram) and the results of several calculations. Ce-123 decays via $\beta$-delayed proton emission to the $0^{+}$ ground state and the $2^{+}$and $4^{+}$excited states of the daughter nucleus ${ }_{56}^{122} \mathrm{Ba}$. The measured final state proton branching ratios are: $20 \pm 7 \%\left(0^{+}\right), 68 \pm 7 \%\left(2^{+}\right)$, and 12 $\pm 3 \%\left(4^{+}\right)$; the half-life is $3.8 \pm 0.2 \mathrm{~s}$. Nilsson model calculations predict the spin of 
TABLE 1. New isotopes and decay branches observed at OASIS $\left({ }^{119} \mathrm{Ba}^{\mathrm{a}},{ }^{129} \mathrm{Nd}^{\mathrm{b}},{ }^{131} \mathrm{Nd}\right.$, ${ }^{133} \mathrm{Sm}^{\mathrm{b}}$, and ${ }^{135} \mathrm{Sm}^{\mathrm{d}}$ also studied but not listed below). From left to right: isotope, measured halflife, calculated half-life (see rexl), decay mode ( $\beta p$ and $\beta g$ denote $\beta$-delaycd proton and $y$ emission, respectively), mean energy and energy range of protons, number of $\gamma$ rays observed, cxperimental spin, calculated spin from Ref. 22 , and whether $x$ rays and/or $\gamma$ rays were observed in coincidence with protons.

\begin{tabular}{|c|c|c|c|c|c|c|c|c|}
\hline ISOTOPE & $I_{1 / 2(c \times D)}$ & $I_{1 / 2(c a l c)}$ & MODE & ENERGY & $\underline{\mathbf{N}}_{\gamma}$ & $\underline{I}^{\pi}(\exp )$ & $\mathrm{r}^{\pi}$ (calc) & PHOTONS \\
\hline $120_{\mathrm{La}^{\mathrm{a}}}$ & $2.8(2)$ & $1.8^{\mathrm{c}}$ & $\beta p$ & $3.7[2.1-5.6]$ & - & - & - & $x$ \\
\hline${ }^{122} \mathrm{La}^{\mathrm{a}}$ & $8.7(7)$ & $5.5^{\mathrm{e}}$ & $\beta p$ & $3.4[2.0-4.6]$ & - & - & - & $\mathbf{x}$ \\
\hline${ }^{123} \mathrm{Cc}^{3}$ & $3.8(2)$ & 2.6 & $\beta p$ & $3.6[2.0-5.8]$ & - & $5 / 2$ & $5 / 2^{+}$ & $x, \gamma$ \\
\hline${ }^{125} \mathrm{Ce}^{\mathrm{c}}$ & $10(1)$ & 10 & $\beta p^{f}$ & $3.3[1.7-5.1]$ & 25 & $5 / 2$ & $1 / 2^{+}$ & $x, y$ \\
\hline${ }^{124} \mathrm{Pr}^{\mathrm{c}}$ & $1.2(2)$ & $0.6^{\circ}$ & $\beta p$ & $3.7[2.2-6.0]$ & $\mathbf{1}$ & - & - & $x, y$ \\
\hline $126 \mathrm{Prg}$ & $3.2(6)$ & $2.0^{\mathrm{e}}$ & $\beta p$ & $3.8[2.1-5.0]$ & - & - & - & - \\
\hline${ }^{128} \mathrm{Pr}^{\mathrm{b}}$ & $3.2(5)$ & $7.7^{\mathrm{e}}$ & $\beta p$ & $3.2[1.9-5.0]$ & - & - & - & $x$ \\
\hline${ }^{127} \mathrm{Nd}^{\mathrm{c}}$ & $1.8(4)$ & 1.7 & $\beta p$ & $3.7[2.2-6.0]$ & - & - & $1 / 2^{+}$ & $x, \gamma$ \\
\hline${ }^{130} \mathrm{Pm}^{\mathrm{b}}$ & $2.2(6)$ & $1.7^{c}$ & $\beta p$ & $3.9[2.1-5.8]$ & - & - & - & $x$ \\
\hline${ }^{132} \mathrm{Pm}^{\mathrm{b}}$ & $5.0(8)$ & $3.7^{\mathrm{c}}$ & $\beta p^{\Upsilon}$ & $3.6[2.2-5.0]$ & - & - & - & $x$ \\
\hline $131 \mathrm{sm}^{\mathrm{c}}$ & $1.2(2)$ & 1.6 & $\beta p$ & $3.7[1.8-6.6]$ & - & - & $7 / 2^{-}$ & $x, y$ \\
\hline${ }^{134} \mathrm{Eu}^{\mathrm{d}}$ & $0.5(2)$ & $1.3^{\mathrm{C}}$ & $\beta p$ & $3.8[1.8-6.0]$ & - & - & - & $x$ \\
\hline $135 \mathrm{Eu}^{\mathrm{d}}$ & $1.5(2)$ & 2.1 & By & n.a. & 1 & - & $1 / 2^{-}$ & n.a. \\
\hline${ }^{136} \mathrm{Eu}^{\mathrm{d}}$ & $3.2(5)$ & $1.8^{\mathrm{e}}$ & $\beta p$ & $3.9[2.0-6.0]$ & 28 & - & - & $x$ \\
\hline${ }^{137} \mathrm{Gdg}$ & $7(3)$ & 1.2 & $\beta p$ & $3.8[2.2-6.6]$ & - & - & $9 / 2^{-}$ & - \\
\hline${ }^{139} \mathrm{Gdg}$ & $S(1)$ & 4.5 & $\beta p$ & $3.8[2.1-5.5]$ & - & - & $7 / 2^{+}$ & - \\
\hline $140 \mathrm{Gd}^{\mathrm{c}}$ & $15.8(4)$ & 15.3 & BY & n.a. & 35 & $0^{+}$ & $\mathrm{D}^{+}$ & л.a. \\
\hline $141 \mathrm{mGd}^{\mathrm{c}}$ & $24.5(9)$ & n.a. & $\beta \gamma$ & n.a. & 35 & $11 / 2$ & n.a. & n.a. \\
\hline $141 \mathrm{BGd}^{\mathrm{C}}$ & $\sim 20$ & 16.3 & $\beta p$ & $3.6[2.2-4.9]$ & 10 & $1 / 2^{+}$ & $1 / 2^{+}$ & $x, y$ \\
\hline $140 \mathrm{~m}^{\mathrm{Tb}} \mathrm{c}$ & $2.4(2)$ & n.a. & $\beta p$ & $4.2[2.2-6.6]$ & 3 & - & л.а. & $x$ \\
\hline${ }^{141} \mathrm{~Tb}^{\mathrm{c}}$ & $3.5(2)$ & 3.4 & $\beta \gamma$ & n.a. & 35 & - & $1 / 2^{-}$ & n.a. \\
\hline $142 \mathrm{~m} \mathrm{~Tb}^{\mathrm{c}}$ & $0.3(1)$ & n.a. & $\beta \gamma$ & n.a. & 4 & - & n.a. & n.a. \\
\hline $142 \mathrm{BTb}^{\mathrm{c}}$ & $0.6(2)$ & $3.5^{\mathrm{e}}$ & $\beta p$ & $3.9[2.5-5.2]^{\mathrm{h}}$ & 7 & - & - & $x, \gamma$ \\
\hline${ }^{141} \mathrm{Dy}^{\mathrm{c}}$ & $0.9(2)$ & 1.4 & $\beta p$ & $4.0[2.4-6.1]$ & - & - & $7 / 2^{+}$ & $x, y$ \\
\hline 142Dyc & $2.3(3)$ & 2.7 & $\beta p$ & $3.9[2.5-5.2]^{\mathrm{h}}$ & 1 & $0^{+}$ & $0^{+}$ & $x, y$ \\
\hline${ }^{143} \mathrm{Dy}^{\mathrm{a}}$ & $3.8(6)$ & 2.9 & $\beta p$ & $4.2[2.5-6.5]$ & - & - & $3 / 2^{-}$ & $x, y$ \\
\hline${ }^{144} \mathrm{Dy}^{\mathrm{C}}$ & $9.1(5)$ & 9.7 & $\beta p$ & $3.2[2.6-4.5]$ & 21 & $0^{+}$ & $0^{+}$ & $x$ \\
\hline${ }^{144} \mathrm{Ho}^{\mathrm{c}}$ & $0.7(1)$ & $1.3^{\circ}$ & $\mathrm{Bp}$ & $4.2[2.2-7.0]$ & - & - & - & $x$ \\
\hline${ }^{146} \mathrm{Ho}^{\mathrm{c}}$ & $3.1(5)$ & $2.9^{\mathrm{e}}$ & $\beta p^{f}$ & $4.1[2.4-6.3]$ & 9 & $\geq 6$ & - & $x$ \\
\hline${ }^{148} \mathrm{~m}_{\mathrm{Ho}} \mathrm{o}^{\mathrm{i}, \mathrm{j}}$ & $9.7(3)$ & n.a. & $\beta p^{f}$ & $4.1[2.2-5.4]^{\mathrm{h}}$ & 39 & $6^{\circ}$ & n.a. & $x, y$ \\
\hline${ }^{148} \mathrm{Er}^{\mathrm{i}}$ & $4.4(2)$ & n.a. & $\beta p^{\mathfrak{l}}$ & $4.1[2.2-5.4]^{\mathrm{h}}$ & 5 & $0^{+}$ & $0^{+}$ & $x, \gamma$ \\
\hline $149 \mathrm{Tm}^{\mathrm{k}}$ & $0.9(2)$ & n.a. & Bp & n.a. & 7 & $1 \mathrm{t} / \mathrm{R}^{-}$ & $112^{-}$ & $x$ \\
\hline $150 \mathrm{mTm}^{\mathrm{i}, \mathrm{j}}$ & $2.2(2)$ & n.a. & $\beta p^{f}$ & $4.7[2.2-7.5]$ & 12 & $6^{-}$ & n.a. & $x, \gamma$ \\
\hline $151 \mathrm{~m} \mathrm{Yb}^{\mathrm{l}}$ & $1.6(1)$ & n.a. & $\beta_{p}$ & $4.0[2.5-6.0]$ & 1 & $11 / 2$ & n.a. & $x, y$ \\
\hline I5lgYbl. & $1.6(1)$ & 0.9 & $\beta p$ & $4.8[2.3-7.5]$ & - & $1 / 2^{+}$ & $112^{2}$ & $x, \gamma$ \\
\hline $152 \mathrm{mLu}^{\mathrm{i}, \mathrm{j}}$ & $0.7(1)$ & n.a. & $\beta p$ & $4.6[2.3-7.9]$ & 3 & $4^{-}, 5^{\circ}, 6^{\circ}$ & n.a. & $x$ \\
\hline
\end{tabular}

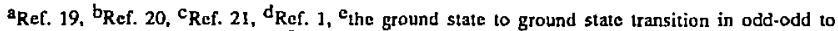
even-even decay is nor included, $\Gamma_{\text {new delayed-proton branch in known isotope, }}$ BRef. $23, h_{\text {mixlure }}$ of all delayed protons in this mass chain, ${ }^{i}$ Rer, $5, j_{\text {relative position of low- and high-spin isomers }}$ unlonown. ${ }^{k}$ Ref. $24,{ }^{1}$ Ref. 12. 
${ }^{123} \mathrm{Ce}$ to be $5 / 2^{+}$(with a $5 / 2^{-}$state in close proximity). Fig. 2 shows the result of an $S_{\beta}$ calculation, described in section 1 , and the $\beta$ intensity distribution $I_{\beta}$. The $\beta$ strength function combined with a statistical model calculation generates four proton spectra representing transitions to the ground and the first two excited states in ${ }_{56}^{122} \mathrm{Ba}$ and their sum, labeled $0^{+}, 2^{+}, 4^{+}$, and "SUM." The precursor spin most consistent with the experimental results is $5 / 2^{+}$which gives final state proton branching ratios of $17 \%$ $\left(0^{+}\right), 73 \%\left(2^{+}\right)$, and $10 \%\left(4^{+}\right)$. Other spin/parity assumptions give proton branching ratios that are at variance with the experiment. The calculated half-life obtained by integrating $I_{\beta}$ (Fig. 2) from 0 to $Q_{E C}$ is $2.6 \mathrm{~s}$. An empirical quenching factor of 0.5 was applied to the $\beta$ strength. The other half-lives in Table 1 were obtained in the same fashion, with $Q_{E C}$ values taken from Ref. 11. The main purpose of presenting the ${ }^{123} \mathrm{Ce}$ example is to point out that $S_{\beta}$ acts as an "anchor" for the proton spectrum. The shape of the spectrum is sensitively dependent on $S_{\beta}$. In the absence of erratic variations in $\Gamma_{\gamma}$ it may even be used to judge whether its shape in the energy range spanned by the proton spectrum is correct. $A$ constant $S_{\beta}$ for example will in general reproduce neither the correct width nor the centroid of the proton spectrum; neither will variations in the other input parameters to the statistical model.

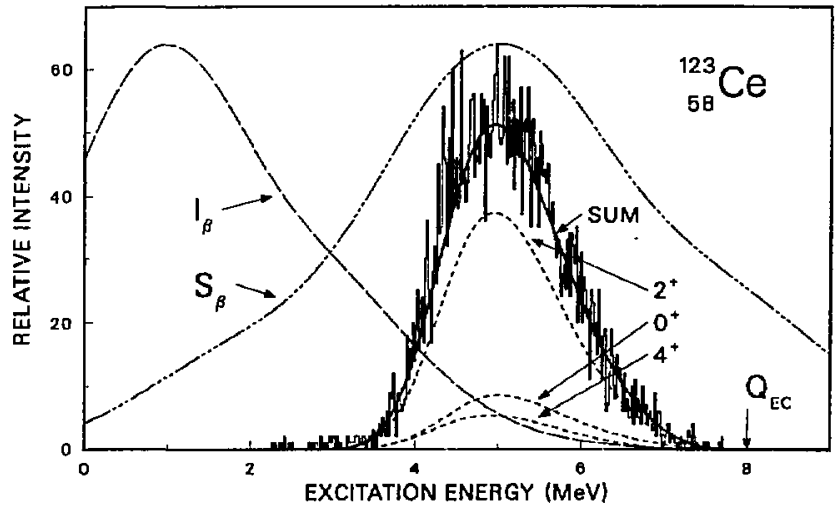

Fig. 2. Experimental proton spectrum (histogram). Proton spectra calculated with a statistical model (solid line and short dashed iines), and $\beta$ - strength function $\mathrm{S}_{\beta}$ (dash dots). Beta intensity $I_{\beta}$ (long dashes) as used for half-life cylculation.

\section{RESULTS: CLOSED SHELL REGION}

In the deformed region the statistical model coupled with $S_{\beta}$ calculations based on Nilsson model wave functions gives an adequate description of $\beta$-delayed proton decay. It is reasonable to expect that the statistical treatment will break down near shell closures because of low level densities. Another dificulty is that rapidly changing deformations will cause the $\beta$ decay mother and daughter to have slightly differing basis wave functions contrary to what is assumed in the calculation of $S_{\beta}{ }^{7}$. To quantify the influence of closed shells on the $\beta$-delayed proton decay we have performed a systematic. study of $\operatorname{six} \mathrm{N}=81$ proton precursors: ${ }^{147} \mathrm{Dy},{ }^{148} \mathrm{Ho},{ }^{149} \mathrm{Er},{ }^{156} \mathrm{Tm},{ }^{151} \mathrm{Yb}$, and ${ }^{152} \mathrm{Lu}$. The observed proton spectra as functions of excitation energy are shown in Fig. 3 (other results are summarized in Table 1 ). 
Fig. 3. Beta-delayed proton spectra of $\mathrm{N}=$ 81 precursors. Proton scparation energies were taken from Ref. 25 and $Q_{E C}$ values from Ref. 11.

Castial inspection of Fig. 3 reveals two prominent effects: (1) an odd-even effect in the mean proton energies and (2) pronounced structure in the spectra originating from cven-odd precursors. The first effect is easily explained by the fact that in oddodd precursors GT $\beta$ decay occurs without breaking a proton pair which leaves the proton emitting nucleus in a state of high excitation energy and consequently high level densities which is also the reason for the statistical nature of the spectra. The average difference between the mean proton energies of the odd-odd and even-odd spectra is $\mathbf{1 . 7}$ $\mathrm{MeV}$ which is expected since the pairing gap js $\Delta=12 / \sqrt{A}$, and thus $2 \Delta$ is $\sim 2 \mathrm{MeV}$ in this region. Details of the decay of oid-odd precursors are discussed in Ref. 5.

It was realized early on that the structure in the even $\mathrm{Z}, \mathrm{N}=81$ precursor spectra may be related to the low level densities of $\mathrm{N}=82$ proton emitters. With the discovery of ${ }^{151} \mathrm{Y} b$ and our ability to separate the spectra into a structured and a statistical component ${ }^{12}$ a more complete picture of the proton decay has emerged with the following features. The proton spectra of the even-odd precursors (Fig. 3b, $d$, and $f$ ) are superpositions of two components, one originating from the $\beta$ decay of the $h_{1 / 2}$ isomeric state (unstructured), and the other associated with the decay of the $B_{1 / 2}$ ground state (highly structured). The decay of the isomer samples high spins, high level densities and high excitation energies in the intermediate nucleus, and the protons are emitted to final states of large angular momenta which subsequently decay by $\gamma$ emission. The favored decay path of the $s_{1 / 2}$ precursor ground state, on the other hand, leads to low spin, Jow level density, and low excitation energy states in the intermediate nucleus.

Statistical model calculations show that the proton decay from these states proceeds with greater than $95 \%$ probability to the $0^{+}$ground state of the final nucleus. The fact that the structure in the proton spectia persists in ${ }^{151} \mathrm{Yb}$ indicates that the $N=82$ rather than the $Z=64$ shell closure is the relevant condition for the effect. 


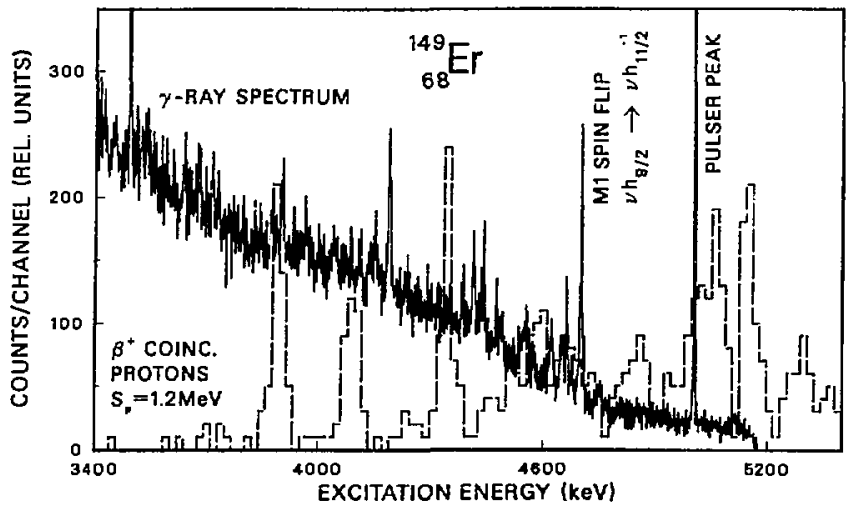

Fig. 4. Positron coincident proton spectrum and $\gamma$-ray singles spectrum following $\beta$ decay of ${ }_{68}^{149} \mathrm{Er}$. $S_{p}$ was taken from Ref. 13.

To elucidate the nature of the proton emitting states in ${ }^{149} \mathrm{Ho}$ we have measured the ligh energy $\gamma$-ray spectrum of ${ }^{149} \mathrm{Er}$ (Fig. 4). The spectrum ends abruptly with the $h_{8 / 2} \longrightarrow h_{11 / 2}^{-1}$ spin flip transitions, the most prominent being at $4.70 \mathrm{MeV}$, while the last strong proton "peak" occurs at $5.14 \mathrm{MeV}$. Similar observations were made for ${ }^{147} \mathrm{Dy}{ }^{13}$. In both cases no obvious correlations between the $\gamma$-ray and proton spectra exist.

In the following an interpretation of the structured proton spectra is proposed based on the concept of doorway states (for a review on this subject see, for example, Ref. 14). The concept was originally introduced to calculate neutron resonances and strength functions ${ }^{15}$ and later generalized for other nuclear reactions. The basic idea can best be described with the simple example of a single nucleon from the continuum interacting with a closed shell target nucleus (say, ${ }^{146} \mathrm{Gd}+\mathrm{p} \longrightarrow{ }^{147} \mathrm{Dy}$ ) [one particle configuration (1p0h)]. If it is assumed that the residual interaction between the incident nucleon and the target nucleons is a sum of two body potentials only two particle- one hole (2p1h) configurations can be excited. These are called "doorway" states because they exhaust most of the coupling strength to a channel in a given range of excitation energies: they are the doorways to most subsequent forms of excitations of the nucleus. The width of these states is $\Gamma=\Gamma \uparrow+\Gamma \downarrow$ where $\Gamma \uparrow$ is the escape width back to the entrance channel and $\Gamma \downarrow$ the damping or spreading width to the compound nucleus.

When treated in first order perturbation theory the probability for transitions from the doorway state with p particles and $h$ holes to states of next higher complexity with $\mathrm{p}^{\prime}$ particles and $h^{\prime}$ holes can be written as

$$
\lambda_{p h \rightarrow p^{\prime} h^{\prime}}=\frac{2 \pi}{\hbar}\left\langle|M|^{2}\right\rangle \rho_{p^{\prime} h^{\prime}}
$$

where $\left\langle|M|^{2}>\right.$ is a suitably averaged matrix element and $\rho_{p^{\prime} h^{\prime}}$ is the density of $p^{\prime}$ particle $h^{\prime}$ hole states. For a simple uniform spacing model Ericson ${ }^{16}$ has given a formula for the density of particle hole states:

$$
\rho_{p h}=\frac{g(g U)^{p+h-1}}{p ! h !(p+h-1) !}
$$


where $U$ is the exeitation energy of the nucleus and $g$ the density of single particle states.

Fig. 5. Schematic shell model representation of the $\beta$-delayed proton and $\gamma$ decay of an even $Z$, $\mathrm{N}=81$ isotone. The shadowed boxes symbolize "doorway" states (see text). The question mark in the center box indicates that the nuclear structure of
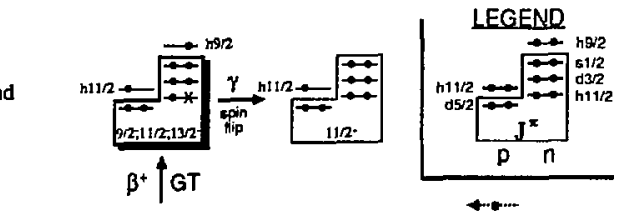
this state(s) is unknown.

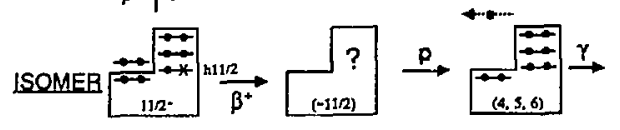
unknown.

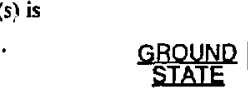
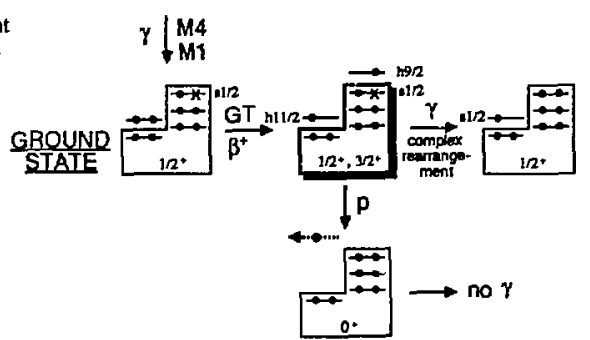

Thus far it was assumed that the (2plh) doorway states are excited through the residual interaction between an incident particle and the target nucleons mediated by two body potentirls. It will now be argued that similar states can be excited in $\beta$ decay and may under certain conditions, become manifest as structure in the subsequent proton decay. This is demonstrated by referring to a simple shell model picture (Fig. 5) which shows the major decay mades of the isomer and the ground state for a typical even- $Z, N=81$ proton precursor (say, ${ }^{147} D y$ ). The decay is dominated by $\pi h_{11 / 2} \longrightarrow \nu h_{9 / 2}$ GT transitions which change the (0p1h) configurations to (2p1h) excited states. Depending on the orbital of the spectator neutron, high or low spin states are produced that have $\left(\pi h_{11 / 2} \nu h_{9 / 2}\right) \times \nu h_{11 / 2}^{-1}$ or $\left(\pi h_{11 / 2} \nu h_{9 / 2}\right)$ $x \nu s_{1 / 2}^{-1}$ configurations respectively. By definition both configurations are docrway states (shadowed boxes in Fig. 5) because all further excitations of the nucleus have to go through them. The channels into which the two "doorway isomers" decay are, however, distinctly different. Measurements of $S_{\xi}$ for ${ }^{147 m} \mathrm{Dy}^{6}$ show a strong GT resonance at $4.7 \mathrm{MeV}$. The width of the resonance is determined by the coupling of the $h_{11 / 2}$ proton and $h_{9 / 2}$ neutron, and the coupling of this pair to different proton configurations, compatible with GT selection rules. Most of these states $\gamma$ decay via a $\nu_{9 / 2} \longrightarrow \nu_{1 / 2}^{-1} \mathrm{Ml}$ spin fip transition in $\sim 10^{-16} \mathrm{~s}^{17}$ to the ground state which has been clearly observed in the spectra of ${ }^{147} \mathrm{Dy}^{13}$ and ${ }^{149} \mathrm{Er}$ ( $\mathrm{Fig}$. 4). In contrast, proton decay to the even-even daughter nucleus from $5 \mathrm{MeV}$ excitation is too weak to be observed either due to the large $\left(9 / 2^{-}, 11 / 2^{-}, 13 / 2^{-}\right) \rightarrow 0^{+}$angular momentum hindrance for decay to the ground state or the small available decay energy to the high lying high spin excited states. The unstructured $\beta$-delayed proton decay from the $h_{11 / 2}$ precursor in ${ }^{149} \mathrm{Er}$ and ${ }^{151} \mathrm{Yb}$ is therefore due to, as yet unknown, configurations at greater than 
$5 \mathrm{MeV}$ excitation energy which have sufficiently small widths $\left(\hbar / \Gamma_{\gamma} \geq 10^{-14} \mathrm{~s}\right)(\mathrm{Fig} .5$ second row). For ${ }^{147 m}$ Dy no significant $\beta$-delayed proton decay is expected. Statistical model calculations using $S_{\beta}$ from Ref. 6 give a branching ratio of $<2 \times 10^{-6}$, mostly due to high $\ell$-wave protons. This corresponds to $<0.4 \%$ of the ${ }^{147} \mathrm{Dy}$ delayed proton branch reported in Ref. 18.

In contrast to the $\gamma$ decay of the high spin doorway state the $\gamma$ decay from $1 / 2^{+}$and $3 / 2^{+}(2 \mathrm{p} 1 \mathrm{~h})$ states proceeds only very slowly, terminating in low-lying proton states which necessitates a complex rearrangement of the nucleus (Fig. 5 third row). This rearrangement requices a large number of nucleon-nucleon interactions. According to eqs. (1) and (2) the spreading width $\Gamma \downarrow$ is strongly dependent on the level density and excitation energy; for (2p1h) states it is proportional to $g(g U)^{p+h-1}=g(g U)^{3+2-1}=g^{5} U^{4}$. The low level density of the closed shall intermediate nucleus and its low excitation energy in the region of the observed structured proton decay $(\sim 5 \mathrm{MeV}$ ) therefore result in a small value of $\Gamma \downarrow$ which retards the $\gamma$ decay of the (2p1h) states. These "simple" states couple, however, very effectively to the open channel wave function and thus become manifest as structure in the proton spectra.

To obtain the relevant matrix elements for GT decay to (2p1h) states we have carried out Nilsson model calculations with a modified oscillator potential as described in section 1. For these calculations it was necessary to introduce "effective" $\varepsilon_{2}$ and $\epsilon_{4}$ deformations of the nucleus, larger than the static ground state deformations. This has two effects: (1) it shifts the GT resonances to lower energies and (2) it distributes their strength over several smaller regonances. For ${ }^{151} \mathrm{Yb}$ for example the ground state deformations ${ }^{9} \varepsilon_{2}=-0.091, \varepsilon_{4}=0.015$, and $\varepsilon_{6}=0.002$ were replaced by $\tilde{\varepsilon}_{2}=$ $-0.150, \tilde{\varepsilon}_{4}=0.040$, and $\tilde{\varepsilon}_{6}=G$. Since the potential mass/energy surface in this region is quite soft this corresponds to a change in ground state energy of only $\sim 500 \mathrm{keV}$. This treatment may also be justified by the observaticn that the proton emission proceeds from elevated excitation energies that are in general associated with larger deformations. Fig. 6 shows the results of the $\mathrm{S}_{\beta}^{G T}$ calculations (c), the calculated (b), and the experimental (a) proton spectra for the three even-odd $N=81$ precursors. Due to limitations in computer time, only a modest effort was made to vary the $\varepsilon_{2}$ and $\varepsilon_{4}$ deformation parameters to match the experiment. The strength of the residual interaction was kept constant and $\varepsilon_{6}$ equal to zero. The calculations reproduce three basic features of the experimental results: (1) the location of the $\beta$ strength, (2) the splitting into smaller resonances. and (3) their average spacing. This is taken as a strong indication that the calculated matrix elements represent the doorway states that are subsequently observed in the proton decay.

\section{CONCLUSIONS AND ACKNOWLEDGMENTS}

A large number of $\beta$-delayeci proton emitters in the region bounded by $Z>50$ and $\mathbf{N}<\mathbf{8 2}$ has now been studied. Beta-delayed proton emission becomes, as expected, a prominent decay mode for all elements near the proton drip line. A significant advance in the understanding of this decay mode has been made by combining a statistical model with $\beta$-strength function calculations based on Nilsson model wave functions in a moditied oscillator potential. The deformation parameters are taken from calculations that employ a folded Yukawa potential, and in the future it may be more consistent to use the same potential for the $S_{\beta}$ calculation. Eventually the calculations will have to be extended to isomeric states since we have observed several cases of $\beta$-delayed proton decay from such states. A direct measurement of $S_{\beta}$ - for example via total absorption $\gamma$ ray apectrometry - will be necessary to gain further confidence in the calculations and to obtain quantitative information about the spreading width of the initial GT states; presently, this is an adjustable parameter (typically $\sim 0.7 \mathrm{MeV}$ in the deformed region). 

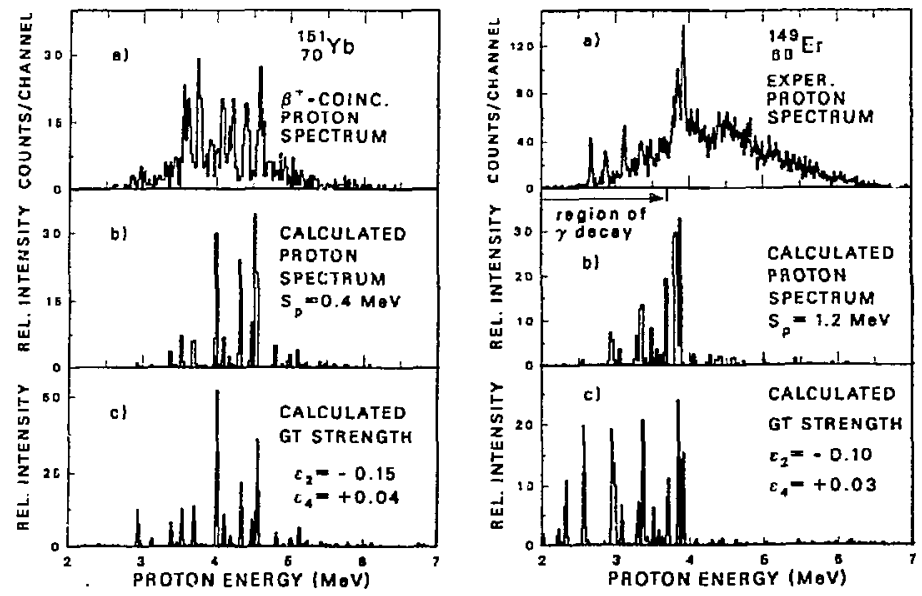

Fig. 6. (a) Beta-delayed proton spectra of even-Z, N $=81$ proton precursors; (b) calculated proton spectra using the $\beta$-strength distribution shown in (c); (c) calculated $\beta$ strength distribution (see text).

For even-Z nuclei near the $N=82$ closed shell pronounced structure in the proton spectra has been cbserved that cannot be treated in the framework of a statistical model. An interpretation based on the concept of doorway states is proposed. Quantitative calculations of these states were carried out that reproduce the essential features of the experimental results.

The participation of $P$. Lemmertz in earlier experiments is appreciated. Valuable comments regarding the shell model were made by R.B. Firestone. F.T. Avignone III and Y.A. Ellis-Akovali participated in the data acquisition phase of several experiments and $K$. Vierinen helped in the preparation of the figures and the $\operatorname{T}_{\mathrm{D}} \mathrm{X}$ version of the manuscript.

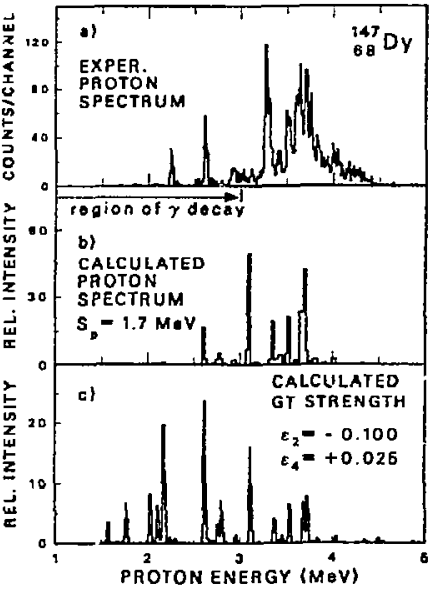




\section{REFERENCES}

1. K. Vierinen, et al., LBL-23221 (to be published) and this conference.

2. P. Hornshøj, et al., Nucl. Phys. A187, 609 (1972).

3. J. Hardy, Phys. Lett. 109B, 242 (1982).

4. C.H. Johnson, et al., Phys. Rev, C20,2052 (1979).

5. J.M. Nitschke, et al., LBL-23408, submitted to Phys. Rev. C.

6. G.D. Alkhazov, et al., Nucl. Phyz. A438, 482 (1985).

7. J. Krumlinde and P. Möller, Nucl. Phys. A417, 119 (1984).

8. S.G. Nilsson, et al., Nucl. Phys. A131, 1 (1969).

9. P. Möller and J.R. Nix, At. Data Nucl Data Tables 26, 165 (1981).

10. J.M. Nitschke, Nucl. Instr. Meth. 206, 341 (1983).

11. S. Liran and N. Zeldes, At. Data Nucl. Data Tables 17, 431 (1976).

12. K.S. Toth, et al., Phys. Lett. B178, 150, (1986).

13. D. Schardt, et al., Proc. 7uh Intl. Corif. Atomic Masses and Fundamental Coristants (AMCO7), O. Klepper (ed.) (Darmstadt, 198s)p. 222.

14. C. Mahaux, Ann. Rev. Nucl. Sci. 23,193 (1973).

15. B. Block and H. Feshbach, Ann. Phys. 23, 47 (1963).

16. T.E. Erieson, Adv. Phys. 9, 425 (1960).

17. J.M. Blatt and V.F. Weisskopf, "Theoretical Nuclear Physics" (John Wiley and Sons, New York, 1955) p.627.

18. K.S. Toth, et al., Phys. Rev. C30, 712 (1984).

19. J.M. Nitschke, et al., Z. Phys. A916. 249 (1984).

20. P.A. Wilmarth, et al., Z. Plyys. $\mathbf{A 3 2 1}, 179$ (1985).

21. P.A. Wilmarth, et al., Z. Phys. A595, 485 (1986).

22. P.A. Seeger and W.M. Howard, Nucl. Phys. A238, 491 (1975).

23. J.M. Nitschke, et al., Z. Phys. A312, 256 (1983).

24. K.S.Toth, et al., Phys. Rev. C36, 826 (1987)

25. A.H. Wapstra and G. Audi, Nucl. Phys. A432, 55 (1985).

\section{DISCLAIMER}

This report was prepared as an account of work sponsored by an agency of the United States Government. Neither the United States Government nor any agency thereor, nor any of their employes, makes any warranty, express or implied, or assumers any legal liability or responsibility for the aceuracy, cempleteness, or usefulness of any information, apparalus, product, or process disclosed, or represents that its use wou!d not infringe privately owned rights. Reference herein to any specific commercial product, process, or service by trade name, trademark, manufacturer, or otherwise does not necessarily constitute or impiy its endorsement, recommendation, or favoring by the United States Government or any agency therest. The views and opinions of authors expressed herein do not necessarily state or rellect those of the United States Government or any agency thereor. 